\title{
RETOUR(S) DE L'AUTEUR ET « POSTURES LITTÉRAIRES » À L'AUBE DU TROISIÈME MILLÉNAIRE
}

\author{
Eva Voldřichová Beránková
}

\begin{abstract}
The term autor has become the object of growing contempt of many French writers (Flaubert, Mallarmé, Valéry, Proust), literary critics (Thibaudet, Bakhtin, Barthes, Blanchot) and philosophers (Foucault, Deleuze) within the last 120 years. Nevertheless, this trend starts to turn slow in 1980s, owing to weakening of previous dominant position of structuralism. The article makes a brief historical survey of refusing author's instance, then it deals with its gradual regeneration since 1980s until now in detail. An analysis of the term "literary posture", promoted by a Swiss critic Jérôme Meizoz, and its potential methodological use follows for better explanation of this present-day "author's comeback". Does it mean that a new "critical school" is being born in Europe?

Keywords: French literature; literary criticism; death of the author; literary posture; Jérôme Meizoz.

Résumé : Pendant plus d'un siècle, la notion d'auteur a fait l'objet d'une méfiance grandissante de la part d'un bon nombre d'écrivains (Flaubert, Mallarmé, Valéry, Proust), critiques littéraires (Thibaudet, Bakhtine, Barthes, Blanchot) et philosophes français (Foucault, Deleuze). Or, depuis les années 1980, parallèlement à un certain affaiblissement de la position dominante du structuralisme, la tendance commence à se renverser. Après avoir résumé l'histoire du rejet de l'auteur, l'article suit sa réhabilitation progressive des années 1980 à nos jours. Pour mieux illustrer l'actuel « retour de l'auteur », il analyse la notion de " posture littéraire », lancée par le critique suisse Jérôme Meizoz, et ses impacts possibles sur la méthodologie. Assistons-nous à la naissance d'une nouvelle « école critique » ?
\end{abstract}

Mots clés : littérature française ; critique littéraire ; mort de l'auteur ; posture littéraire ; Jérôme Meizoz.

\section{L'auteur en disgrâce}

Pendant plus d'un siècle, la notion d'auteur a fait l'objet d'une méfiance grandissante de la part d'un bon nombre d'écrivains, critiques littéraires et philosophes français. Cette tendance moderne remonterait symboliquement à Flaubert, partisan de «l'écriture impersonnelle », pour qui l'expression littéraire de ses propres idées et sentiments rimait avec vulgarité :

« J'éclate de colère et d'indignations rentrées. Mais dans l'idéal que j'ai de l'art, je crois qu'on ne doit rien montrer des siennes, et que l'artiste ne doit plus apparaître dans son œuvre que Dieu dans la nature. L'homme n'est rien, l'œuvre tout! Cette discipline, qui peut partir d'un point de vue faux, n'est pas facile à observer. Et pour moi, du moins, c'est une sorte de sacrifice permanent que je fais au bon goût. Il me serait bien agréable 
de dire ce que je pense et de soulager le sieur Gustave Flaubert par des phrases, mais quelle est l'importance du dit sieur? » (Flaubert 1893 : 219-220)

Un scepticisme similaire face à toute entreprise ouvertement autobiographique, voire à toute volonté plus générale de "se dépeindre », marque également les réflexions de Marcel Proust : " Fromentin, Musset, malgré tous leurs dons, parce qu'ils ont voulu laisser leur portrait à la postérité, l'ont peint fort médiocre; encore nous intéressent-ils infiniment même par là, car leur échec est instructif. » (Proust 1919 : 198)

Stéphane Mallarmé va encore plus loin. Non seulement il récuse violemment les fictions de soi, mais il en appelle à une véritable « disparition élocutoire du poète » (Mallarmé 1945: 366), désireux de remplacer dans toutes les œuvres littéraires l'auteur concret par l'initiative propre du langage, anonyme et infinie. Paul Valéry, lui aussi, estime dans Tel quel que « toute œuvre est l'œuvre de bien autre chose qu'un auteur'. » (Valéry 2004 : 629)

Dans le domaine de la critique littéraire, dès Thibaudet, nous percevons la même tendance à privilégier les écritures impersonnelles, jugées plus réussies, car plus sophistiquées et susceptibles de créer l'illusion plutôt que de copier la réalité :

Il est très rare qu'un auteur qui s'expose dans un roman fasse de lui un individu vivant. [...] Des romanciers-nés, comme Flaubert ou Maupassant, ne se déposent pas, mais se transposent et se transforment en Frédéric Moreau et en Bel-Ami. [...] S'ils prennent pour sujet de leur œuvre cette existence réelle, elle se réduit en cendre, elle devient fantôme sous la main qui la touche. Elle a eu sa vie, n'a pas droit à une autre. Le génie du roman fait vivre le possible, il ne fait pas revivre le réel. De chaque coulée, il exige qu'elle soit de source, et vierge. (Thibaudet $1925: 5-6$ )

Mikhaïl Bakhtine insiste, lui aussi, sur une distinction radicale entre l'auteur et le héros (le narrateur) au nom de l'esthétique qui doit prévaloir sur les enjeux éthiques voire idéologiques de l'œuvre :

L'événement esthétique, pour s'accomplir, nécessite deux participants, présuppose deux consciences qui ne coïncident pas. Là où le héros et l'auteur coïncident ou bien se situent côte à côte, partageant une valeur commune, ou encore s'opposent en tant qu'adversaires, l'événement esthétique prend fin et c'est l'événement éthique qui prend place (pamphlet, manifeste, réquisitoire, panégyrique et compliment, injure, confession, etc.). (Bakhtine 1984 : 87)

$\mathrm{Si}$, comme nous l'avons vu, l'histoire des polémique est plus ancienne, ce ne sont que les années 1960 qui semblent trancher définitivement avec la traditionnelle «méthode biographique » de Sainte-Beuve et avec «l'histoire littéraire » de Lanson qui, chacune à sa façon, recouraient prioritairement à l'auteur comme principe de cohérence de l'œuvre et qui, en même temps, voiaient en lui une solide figure historique ou psychologique. Résumant en partie certains arguments plus anciens, formulés par les formalistes russes et les structuralistes, Roland Barthes signe son article célèbre dont le titre ne demande rien de moins que «La mort de l'auteur », au nom d'une plus grande liberté, d'une plus grande ouverture du texte :

Nous savons maintenant qu'un texte n'est pas fait d'une ligne de mots, dégageant un sens unique, en quelque sorte théologique (qui serait le «message » de l'Auteur-Dieu), mais un espace à dimensions multiples, où se marient et se contestent des écritures variées, dont aucune n'est originelle : le texte est un tissu de citations, issues des mille foyers de la culture. [...] L'Auteur une fois éloigné, la prétention de « déchiffrer » un texte devient tout à fait inutile. Donner un Auteur à un texte, c'est imposer à ce texte un cran d'arrêt, c'est le pourvoir d'un signifié dernier, c'est fermer l'écriture. (Barthes 1994 : 493-494) 
La même acception des processus langagiers, du « Texte » fonctionnant comme une machine autotélique, indépendante de l'intention ou de la conscience d'un auteur personnel, marque également l'approche critique de Maurice Blanchot : « [...] l'auteur ne doit pas intervenir, parce que le roman est une œuvre d'art et que l'œuvre d'art existe toute seule, chose irréelle, dans le monde hors du monde, il faut la laisser libre, supprimer les détails, couper les amarres, pour la maintenir dans son statut d'objet imaginaire. » (Blanchot 1969: 650)

Dans un autre contexte, les sociologues, eux aussi, œuvrent à libérer le texte d'un auteur individuel. Soucieux de dépasser le sujet et son " projet » singulier, tel que Sartre l'envisageait jadis dans ses critiques de Flaubert ou de Genêt, Pierre Bourdieu lance sa notion de « champ intellectuel » (ayant une dynamique propre et constituant un « espace des possibles ») qui génère plus ou moins nécessairement un certain type de production (Bourdieu 1992). Quant à l'analyse du langage, Roger Chartier propose de remplacer les méthodes axées sur l'auteur par une "pragmatique des discours », destinée à lier le social au linguistique (Chartier 1999 : 276).

Des philosophes tels que Michel Foucault ou Gilles Deleuze s'appliquent, eux aussi, à tirer sur cette notion d'auteur, tantôt dénoncée comme dangereusement réductrice voire répressive, tantôt considérée comme désuète : "Il n'y a pas de littérature sans fabulation, mais, comme Bergson a su le voir, la fabulation, la fonction fabulatrice ne consiste pas à imaginer ni à projeter un moi. »(Deleuze 1999 : 102)

\section{L'auteur réhabilité}

Or, depuis les années 1980, parallèlement à un certain affaiblissement de la position dominante du structuralisme, la tendance se renverse peu à peu et on commence à évoquer timidement un retour possible de différentes notions taboues jusqu'ici, à savoir l' " histoire », le «sujet » voire l' « auteur », sous des formes modifiées ou nouvellement définies.

Des historiens redécouvrent les charmes de la biographie (voir les presque mille pages de Saint Louis de Jacques Le Goff), des théoriciens de la littérature se lancent dans la "critique génétique» (méthode qui suppose un processus créatif individué, dont les stratégies sont recélables dans les divers moments de l'écriture) ou dans la « sociologie des institutions littéraires " (Dubois, Viala, Jurt), tandis que la médiatisation croissante de la littérature fait de l'auteur l'un des principaux « agents de publicité », susceptibles d'influer sur les ventes de l'œuvre-produit.

Certains universitaires vont jusqu'à relativiser les théories (formalisme, structuralisme, Nouvelle critique) en bloc, estimant que dans leur lutte, logique mais somme toute vaine, contre le « bon sens » elle finissent par soutenir des paradoxes intenables (mort de l'auteur, non-existence du lecteur, évacuation de la question de la valeur) dont le radicalisme absurde réduit d'avance toute chance de succès durable :

Certes, l'auteur est mort, la littérature n'a rien à voir avec le monde, la synonymie n'existe pas, toutes les interprétations sont valables, le canon est illégitime, mais on continue à lire des biographies d'écrivains, on s'identifie aux héros des romans, on suit avec curiosité les traces de Raskolnikov dans les rues de Saint-Pétersbourg, on préfère Madame Bovary à Fanny, et Barthes se plongeait délicieusement dans Le Comte de Monte-Cristo avant de s'endormir. C'est pourquoi la théorie ne peut pas remporter. (Compagnon 1998 : 306-307) 
Sans nous prononcer au sujet d'une hypothèse aussi controversée, examinons plus en détail l'une des approches actuelles qui jette une lumière particulière sur ce fameux « retour de l'auteur » dont la communauté littéraire parle de plus en plus, sans toujours bien le cerner sur le plan méthodologique.

\section{Les "postures littéraires"}

Depuis le début des années 2000, Jérôme Meizoz (1967), écrivain et théoricien de la littérature vaudois, travaille sur la notion de "posture littéraire » autour de laquelle une nouvelle tendance critique est en train de se constituer. Héritier de l'approche sociologique de la littérature (Bourdieu notamment, Viala dans une moindre mesure), dont il ne partage pas pour autant tous les postulats, Meizoz propose la définition suivante de son concept fondamental :

Une posture désigne la présentation de soi de l'écrivain, et met donc l'accent sur la construction d'une figure d'auteur singulière par le biais d'un ethos linguistique et de conduites littéraires publiques. Manière singulière d'occuper sa position sérielle dans le champ littéraire, un choix postural ne se comprend qu'en référence à l'espace des possibles du champ. (Meizoz 2011 : 18)

Comme nous pouvons le voir, la posture recouvre deux dimensions à la fois : une approche rhétorique (intratextuelle) et une stratégie actionnelle (extratextuelle), à savoir un discours (qui relève de la rhétorique) et une conduite (qui relève de la sociologie).

Du point de vue textuel, la posture correspond dans une large mesure à la notion rhétorique d'ethos. Afin de pouvoir agir avec efficacité sur son auditoire, l'orateur doit disposer d'arguments valides (maîtriser le logos), produire un effet puissant sur le public (le pathos) et, surtout, "affirmer son autorité et projeter une image de soi susceptible d'inspirer confiance » (Amossy $2004:$ 200-201) (l'ethos).

Pour davantage de précision, nous pouvons constater une légère différence entre les deux notions : tandis que l'ethos est interne à un texte donné, et peut donc changer d'une œuvre à l'autre, la posture reste globalement la même. A titre d'exemple, mentionnons le cas de Rousseau : entre 1762 et 1778, à savoir entre les Lettres à Malesherbes, les Confessions et les Rêveries du promeneur solitaire, ses écrits manifestent plusieurs ethè distincts (d'un auteur inquiet et pressé de se justifier à un stoïcien planant au-dessus de la foule), mais ils relèvent tous de la même " posture » (volonté de déjouer le «complot de ténèbres » qui semble environner le philosophe).

Or, à cette dimension rhétorique (intratextuelle), la posture joint encore tout ce qui est «présentation de soi » voire « auto-création » dans l'espace extratextuel (conduites publiques en situation littéraire : prix, discours, banquets, entretiens, etc.) Autrement dit, la fameuse blouse blanche que Céline a enfilée avant de se présenter aux journalistes lors de la parution du Voyage au bout de la nuit (automne 1932) fait partie de sa " posture auctoriale », au même titre que les procédés stylistiques utilisés dans le texte de son roman.

\section{Les avantages de l'approche posturale}

La théorie de la posture serait, bien sûr, impensable sans la notion d'auteur. Or, il s'agit d'un auteur bien modifié, ayant tiré toutes les conséquences d'un siècle de critique immanente. Meizoz travaille avec les trois «instances auctoriales », définies par le linguiste Dominique Maingueneau (Maingueneau 2004), distinguant entre la personne (su- 
jet biographique et civil : Louis Destouches ou Michel Thomas), l'écrivain (auteur dans le champ littéraire : Céline ou Michel Houellebecq) et l'inscripteur (énonciateur dans le texte : Ferdinand de Mort à crédit ou Michel de Plateforme).

Selon Meizoz « les diverses théories littéraires peuvent se distinguer notamment par l'accent qu'elles mettent sur l'un de ces trois niveaux : d'un côté, les théories d'obédience formaliste réduisent l'instance auctoriale à l'inscripteur ; de l'autre, les théories socio- ou bio-graphiques négligent l'inscripteur, ou tendent à l'identifier sans autre à la personne ou à l'écrivain. La présente réflexion sur la posture, si elle privilégie la figure de l'écrivain, cherche à le penser en relation constante avec la personne et l'inscripteur. » (Meizoz 2007 : 43-44)

En effet, la théorie de la posture représente une tentative intéressante de concilier la critique immanente avec la critique sociologique, l'intratextuel avec l'extratextuel, et par ce même de réhabiliter la notion d'auteur, sans tomber pour autant dans les pièges de la vieille théorie de «l'homme et l'œuvre » ou de l'histoire littéraire lansonienne.

De plus, cette approche présente l'avantage de fournir un outil méthodologique efficace pour étudier certains phénomènes typiques de notre époque. De nombreux auteurs contemporains (Angot, Beigbeder, Nothomb, Donner, Despentes, Houellebecq, etc.) non seulement s'accommodent fort bien de la "culture de masse", mais ils en profitent même, assumant pleinement leur mise en scène publique à travers différentes polémiques portant sur leur personne et leurs écrits. Comment une critique immanente, à savoir strictement limitée aux analyses textuelles, pourrait-elle saisir par exemple tous les jeux houellebecquiens dans lesquels l'écrivain, tour à tour, assume et rejette les convictions de ses propres personnages, fait croire à l'identité de l'auteur et du narrateur d'un roman donné pour la nier aussitôt après, bref, quand il joue à colin maillard avec le public (et les tribunaux), estimant que le procès judiciaire, lui aussi, fait partie l'œuvre?

Si le formalisme et le structuralisme représentaient jadis des réactions salutaires au monopole de la théorie littéraire, ne serait-il pas temps de les reconsidérer à leur tour, non pas pour rejeter leurs apports méthodologiques incontestables, mais pour les élargir à un contexte qui leur fait défaut ? La théorie de la posture littéraire a, entre autres, lancé ce pari logique.

Après un siècle de méfiance voire de négation, l'auteur revient donc au galop. Plus précisément, ils sont désormais trois à revenir.

\section{Bibliographie}

Amossy, Ruth, «Ethos », in : Aron, Paul - Saint-Jacques, Denis - Viala, Alain (2004), Le Dictionnaire du littéraire, Paris : PUF.

BAKHTINE, Mikhail (1984), "L'auteur et le héros dans le processus esthétique », 1920-1930, in : Esthétique de la création verbale, Paris : Gallimard.

BARTHES, Roland (1994), "La mort de l'auteur », in : CEuvres complètes, t. II, Paris : Seuil.

Blanchot, Maurice (1969), « La fin du héros », in : L'Entretien infini, Paris : Gallimard.

BOuRDieu, Pierre (1992), Les règles de l'art : genèse et structure du champ littéraire, Paris : Seuil.

Chartier, Roger (1999), "Cité savante et débat public », in : Le Jeu de la règle, Bordeaux : Presses universitaires de Bordeaux.

ColonnA, Vincent (2004), Autofiction E autres mythomanies littéraires, Auch : Tristram.

Compagnon, Antoine (1998), Le démon de la théorie, Paris : Seuil. 
Deleuze, Gilles (1999), « La littérature et la vie », in : Critique et clinique, Paris : Éditions de Minuit.

Flaubert, Gustave (1893), « Lettre à George Sand de décembre 1875 », in : Correspondance, quatrième série, (1869-1880), Paris : Bibliothèque Charpentier.

Maingueneau, Dominique (2004), Le Discours littéraire. Paratopie et scène d'énonciation, Paris : Armand Colin.

Mallarmé, Stéphane (1945), «Crise de vers », in : CEuvres complètes, Paris : Gallimard, Bibliothèque de la Pléiade.

Meizoz, Jérôme (2007), Postures littéraires. Mises en scène modernes de l'auteur, Genève : Slatkine Érudition.

Meizoz, Jérôme (2011), La fabrique des singularités. Postures littéraires II, Genève : Slatkine Érudition.

Proust, Marcel (1919), « À propos de Dominique et de La Confession d'un enfant du siècle, Journées de lecture », in : Pastiches et mélanges, Paris : Gallimard.

THIBAUdET, Albert (1925), «L'Esthétique du roman », in : Le Liseur de romans, Paris : G. Crès \& Cie.

Valéry, Paul (2004), Tel Quel, in : CEuvres, t. II, Paris : Gallimard, Bibliothèque de la Pléiade.

\author{
Eva Voldřichová Beránková \\ Ústav románských studií \\ Filozofická fakulta \\ Univerzita Karlova v Praze \\ Náměstí Jana Palacha 2 \\ 11000 Praha 1 \\ République tchèque \\ berankova.eva@volny.cz
}

